\title{
Effects of saturation on the forces in induction motors with whirling cage rotor
}

\author{
Asmo Tenhunen, Timo, P. Holopainen*, Antero Arkkio \\ Laboratory of Electromechanics \\ Helsinki University of Technology \\ P.O. Box 3000, FIN-02015 HUT, Finland \\ Asmo.Tenhunen@hut.fi \\ *VTT Industrial Systems \\ Technical Research Centre of Finland \\ P.O. Box 1705, FIN-02044 VTT, Finland \\ Timo.Holopainen@vtt.fi
}

\begin{abstract}
The effects of magnetic saturation on the radial magnetic force in induction machines when the rotor is performing cylindrical circular whirling motion are studied in this paper. Impulse method in the finite element analysis is used to calculate the forces and the eccentricity harmonics from both, the air gap flux density and rotor currents. The forces are studied as a function of supply voltage in order to find out the effects of the saturation on them. The maximum radial force is found to be limited by saturation and saturation also couples the eccentricity harmonics together.
\end{abstract}

\section{INTRODUCTION}

An electromagnetic force acts between the rotor and stator if the rotor is performing cylindrical circular whirling motion with respect to the stator [1]. The magnetic saturation influences on the amplitude and the direction of the force.

The effects of saturation are quite difficult to take into account analytically and the analytical models give only an approximation of the saturation effects [2]. In the numerical studies of rotor eccentricity, some results of the effects of saturation are presented in $[1,3]$.

In this study, the effects of saturation are studied using impulse method in finite element analysis. The forces are calculated as a function of whirling frequency and supply voltage at no load and rated load. The effects are also considered from the air-gap flux density and rotor current harmonics.

\section{METHODS}

The calculation of the magnetic field is based on the timestepping, finite element analysis. The magnetic field is supposed to be two-dimensional and the laminated iron core is treated as a non-conducting magnetically nonlinear medium, which is modeled by a single-valued magnetization curve (Fig. 1). For a comparison, the forces were also calculated for a linearized motor which has a constant relative permeability $\mu_{\mathrm{r}}=1000$.

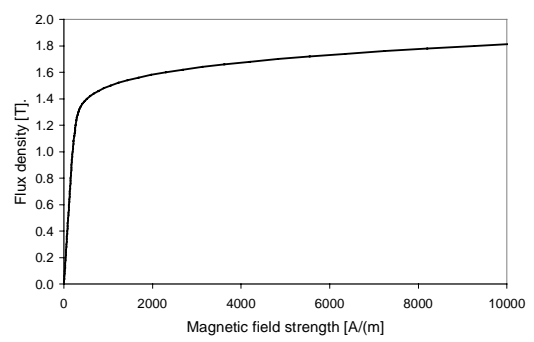

Fig. 1. The single-valued magnetization curve used.

The impulse method [4] is utilized in the analysis. The basic idea of the impulse method is to move the rotor from its central position for a short period of time. The displacement excitation produces the flux density harmonics into the air gap. The harmonics, in their turn, induce the equalizing currents into the rotor bars and cause forces between the rotor and stator. During the time-stepping analysis, the harmonics of order $p \pm 1$ ( $p$ is number of pole pairs of the motor) from rotor currents and air gap flux density distribution are calculated at each time step. The forces are calculated by a method, based on the principle of the virtual work [5]. Using spectral analysis techniques the frequency response of the harmonics and forces is calculated using the excitation and response signals.

\section{RESULTS}

A four-pole $15 \mathrm{~kW}$ induction motor was chosen for test motor. The parameters of the motor are given in [4]. In the analysis, the length of the cosine-type displacement pulse was $0,01 \mathrm{~s}$ and the amplitude $11 \%$ of the air gap length. The total simulation time was $1 \mathrm{~s}$ with a constant time-step of $0.05 \mathrm{~ms}$. In the spectral analysis, the number of sample points was 8192 and the length of the signal $4 \mathrm{~s}$, by adding the zero level at the end of the sample to increase the frequency resolution.
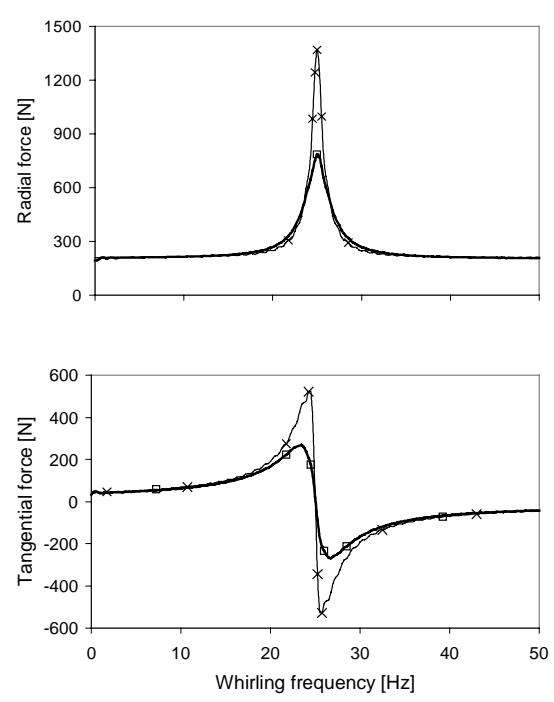

b)

Fig. 2. The radial and tangential component of the force as a function of whirling frequency at no load. $\bigcirc$ - normal and $\mathrm{x}$ - the linearized motor. 
If the forces are divided into a radial component in the direction of the shortest air gap and a tangential component perpendicular to the radial one, the components are almost independent on time.

Fig. 2 shows the radial and tangential components of the force as a function of the whirling frequency at no load and supplied by the rated voltage $380 \mathrm{~V}$ for both normal and linearized motor.

Fig. 3 shows the radial and tangential components of the force as a function of the supply voltage at whirling frequencies $5 \mathrm{~Hz}$ and $25 \mathrm{~Hz}$. At no-load condition, the maximum force occurs at $25 \mathrm{~Hz}$ for the four-pole motors. Fig. 3 shows that the maximum force is reached near to 300 $\mathrm{V}$ and for bigger values of supply voltages the amplitude of the force is decreased. At a $5 \mathrm{~Hz}$ whirling frequency, the flux density harmonics are strongly damped by rotor currents and the radial component of the force behaves like in the linearized case.

The ratio of the tangential and radial force components changes with the voltage. This means that the saturation also affects the direction of the force.
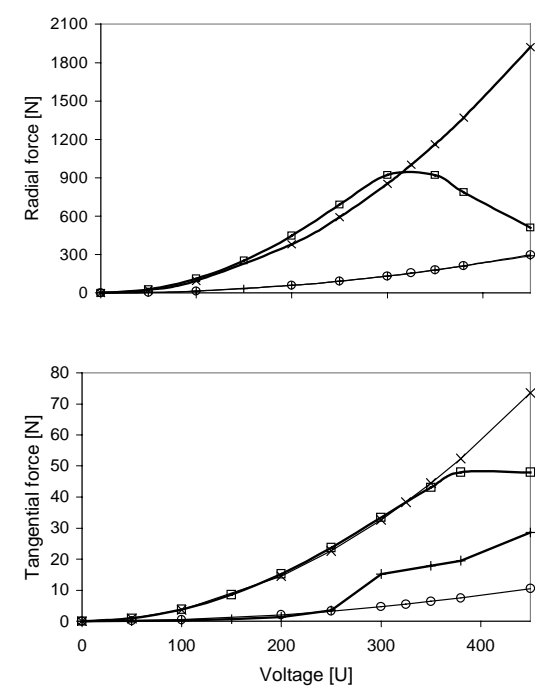

Fig. 3. The amplitudes of the force components as a function of supply voltage at no load. Marking: x - linearized $25 \mathrm{~Hz}, \square-$ normal $25 \mathrm{~Hz}, \circ$ linearized $5 \mathrm{~Hz}$ and + - normal $5 \mathrm{~Hz}$.

Fig. 4 shows the behaviour of the flux density harmonics $p \pm 1$ as a function of supply voltage at whirling frequencies 5 $\mathrm{Hz}$ and $25 \mathrm{~Hz}$ at no load and voltage level $380 \mathrm{~V}$.

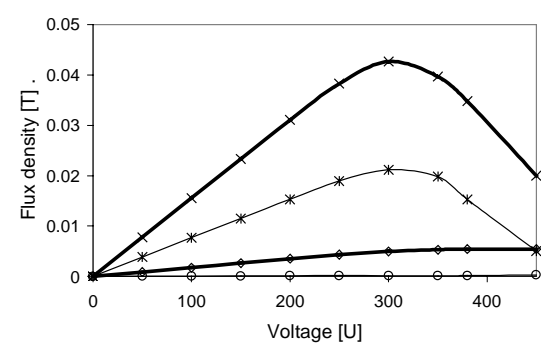

Fig. 4. The flux density harmonics $(p \pm 1)$ as a function of supply voltage at rated load. Marking: $\mathrm{x}-p+1$ at $25 \mathrm{~Hz}, *-p-1$ at $25 \mathrm{~Hz}, \diamond-p+1$ at $5 \mathrm{~Hz}$ and $\circ-p-1$ at $5 \mathrm{~Hz}$
The effects of saturation can be seen easily from the flux density harmonics versus voltage behaviour. The amplitudes of the harmonics start to decrease approximately at the same voltage as the forces.

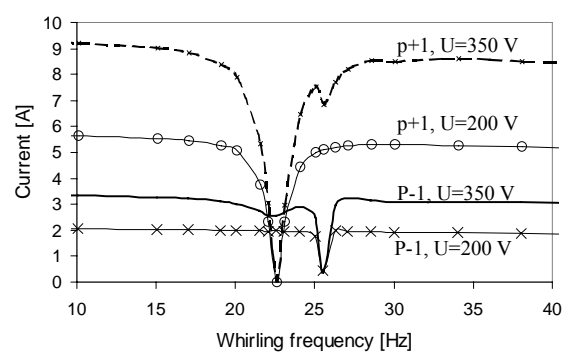

Fig 5. $p \pm 1$ harmonics of the rotor currents at rated load at $350 \mathrm{~V}$ and $200 \mathrm{~V}$.

Until now, we have discussed the results calculated at no load. At rated load, the behavior of the forces and flux density harmonics is similar as at no load, except that the frequency response of the forces has two peaks, because the slip differs from zero at the rated load. Then the maximum force and also the maximum value of flux density harmonics occur at different whirling frequencies. Respectively, the minimum of the rotor current harmonics occurs at different whirling frequencies. Fig. 5 presents the calculated rotor current harmonics $p \pm 1$ as a function of whirling frequency at rated load and at supply voltages $200 \mathrm{~V}$ and $350 \mathrm{~V}$. The coupling of the harmonics due to the saturation can be seen from figure. At $350 \mathrm{~V}$, the motor core is saturated and the current harmonics seem to influence each other. At $200 \mathrm{~V}$, the motor core is not saturated and no coupling can be found.

\section{CONCLUSIONS}

The effects of saturation on the electromagnetic forces in induction machines with rotor eccentricity are studied. The forces and harmonics of flux density and rotor currents are calculated using impulse method in finite element analysis. The results show that the magnetic saturation limits the maximum force and influences also on the direction of the force.

\section{REFERENCES}

[1] A. Arkkio, M. Antila, K. Pokki, A. Simon and E. Lantto, “ Electromagnetic force on a whirling cage rotor", IEE Proc. Elect. Power Appl., vol. 147, pp. 353-360, September 2000.

[2] D. G. Dorrell, "Experimental behaviour of unbalanced magnetic pull in 3-phase induction motors with eccentric rotors and the relationship with tooth saturation", IEEE Transactions on Energy Conversion, vol. 14, pp. 304-309, September 1999.

[3] A. Arkkio and O. Lindgren, "Unbalanced magnetic pull in a highspeed induction motor with an eccentric rotor", Proceedings of ICEM'94, Paris, France, 5-8 September 1994, pp. 53-58.

[4] A. Tenhunen, T. P. Holopainen and A. Arkkio, "Impulse method to calculate the frequency response of the electromagnetic forces on whirling cage rotors", Accepted to be published in IEE Proc. Elect. Power Appl.

[5] J. L. Coulomb, "A methodology for the determination of global electro-mechanical quantities from a finite element analysis and its application to the evaluation of magnetic forces, torques, and stiffness", IEEE Transactions on Magnetics, vol. 19, pp. 2514-2519, November 1983. 